\title{
Ábacos para Pré-dimensionamento de treliças e tesouras de cobertura com perfis formados a frio \\ Cristiano Rossoni ${ }^{1}$, Judiclar Rigo ${ }^{1}$, Marinês Silvani Novello ${ }^{2}$, Zacarias Martin Chamberlain Pravia ${ }^{3 *}$ \\ ${ }^{1}$ Engenharia Civil, Universidade de Passo Fundo, Passo Fundo, biancoross@gmail.com, judiclair_rigo@yahoo.com.br \\ ${ }^{2}$ Engenharia Civil, Faculdade Meridional - Imed, Passo Fundo, RS, marines.novello@imed.edu.br \\ ${ }^{3}$ Programa Pós-Graduação em Engenharia Civil e Ambiental, PPGEng, Universidade de Passo Fundo, Passo Fundo, RS, zacarias@upf.br
}

\section{ABACUSES TO PRE-DESIGN COLD-FORMED STEEL TRUSSES}

\section{Resumo}

O projeto da estrutura de uma cobertura em estrutura de aço treliçada é composta por várias fases, sendo uma delas a de pré-dimensionamento. Na bibliografia técnica somente encontram-se fórmulas empíricas e gráficos que através do vão livre teórico definem apenas a altura da viga treliçada. Diante disso, esse trabalho dá continuidade ao trabalho de BIANCHI, NOVELLO e PRAVIA (2015), agora com treliças de banzos paralelos e tesouras em perfis formados a frio, considerando além do vão livre, premissas como, espaçamento e ações permanentes e variáveis, através de ábacos e tabelas obtêm-se os dimensionais da seção transversal do perfil, mais próximos do real, que podem ser utilizados nos projetos.

Palavras-chave: pré-dimensionamento, treliças de aço.

\section{Abstract}

The design of a truss steel structure roof consists of several stages, after defined the global dimensions need to define sections of elements: pre-design. In literature area founded only empirical formulas and graphics that give global sizing, as relation of span height of truss, but not sections. Therefore, this work continues the presented by BIANCHI, NOVELLO and PRAVIA (2015) and presents tables and abacuses to pre-design elements of planar trusses.

Keywords: presizing, steel trusses.

${ }^{*}$ Autor correspondente 


\section{INTRODUÇÃO}

A elaboração do projeto de uma estrutura é composta por fases de prédimensionamento, análise estrutural, verificação de resistências e estabilidade, bem como verificação dos limites de deformações. Os resultados dessas fases permitem a elaboração do projeto detalhado, sendo que nessas etapas são determinadas as dimensões das seções transversais dos elementos que serão utilizados para formar a estrutura da edificação.

A maioria das publicações existentes considera equações e gráficos empíricos e ou regras decorrentes de práticas aplicadas durante a execução de projetos. Rebello (2007) afirma que as treliças planas mais econômicas são as que apresentam a relação entre altura da treliça e vão compreendido entre $1 / 7$ e 1/10, e em casos extremos podem ser utilizados valores entre $1 / 5$ a $1 / 15$ do vão livre teórico, porém já não sendo tão econômicos. Ocorre que esse pré-dimensionamento se limita às dimensões globais do modelo estrutural (treliças ou tesouras), sem fornecer as dimensões das seções a dispor nos elementos do arranjo estrutural.

\section{JUSTIFICATIVA E OBJETIVOS}

Diante dessa necessidade, e dando continuidade ao trabalho de BIANCHI, NOVELLO e PRAVIA (2015), o qual a partir de modelos definidos de arcos treliçados têm-se a seção do perfil mais próxima da esperada que pode ser aplicada nos projetos, o objetivo deste trabalho também é apresentar as seções de perfis em aço formados a frio porém para elementos em forma de cantoneiras duplas e perfis " $U$ " que compõem vigas treliçadas planas em duas águas do tipo trapezoidais (RIGO, 2014) e retangulares de banzos paralelos (ROSSONI, 2015). Nas verificações foram considerados os perfis mais adequados para atender às solicitações de segurança estrutural e critérios das normas ABNT.

A meta do presente trabalho é determinar com as prescrições de estados limites e de utilização dimensões de seções que possam ser um ponto de partida para prédimensionar e configurar projetos para poder analisar e verificar os elementos de maneira mais eficiente. De maneira secundária, apresentam-se os procedimentos necessários para o cálculo de edificações deste tipo. 


\section{METOdOLOGIA}

A seguir são definidos os processos de dimensionamento dos conjuntos de edificações industriais que foram usadas, as seções que foram consideradas de acordo com a padronização das normas brasileiras e as ações e prescrições seguidas no dimensionamento.

As seções transversais escolhidas foram as seções padronizadas pela ABNT NBR 6355:2012 Nas análises e dimensionamentos apresentados a seguir foram consideradas as seguintes hipóteses:

- sistema estrutural transversal: pórticos com ligações rígidas e bases engastadas;

- sistema estrutural longitudinal: pórticos contidos verticalmente com bases rotuladas;

- treliças de cobertura trapezoidais e retangulares, ambas com contenções laterais a cada dois nós e contenções laterais no banzo superior travados a cada nó da treliça onde são instaladas as terças de cobertura;

- colunas sem contenções laterais;

- perfis com seção de dupla cantoneira e perfil U em aço estrutural ASTM A 572 grau 50 dispostos em diferentes posições conforme figuras 1 e 2.

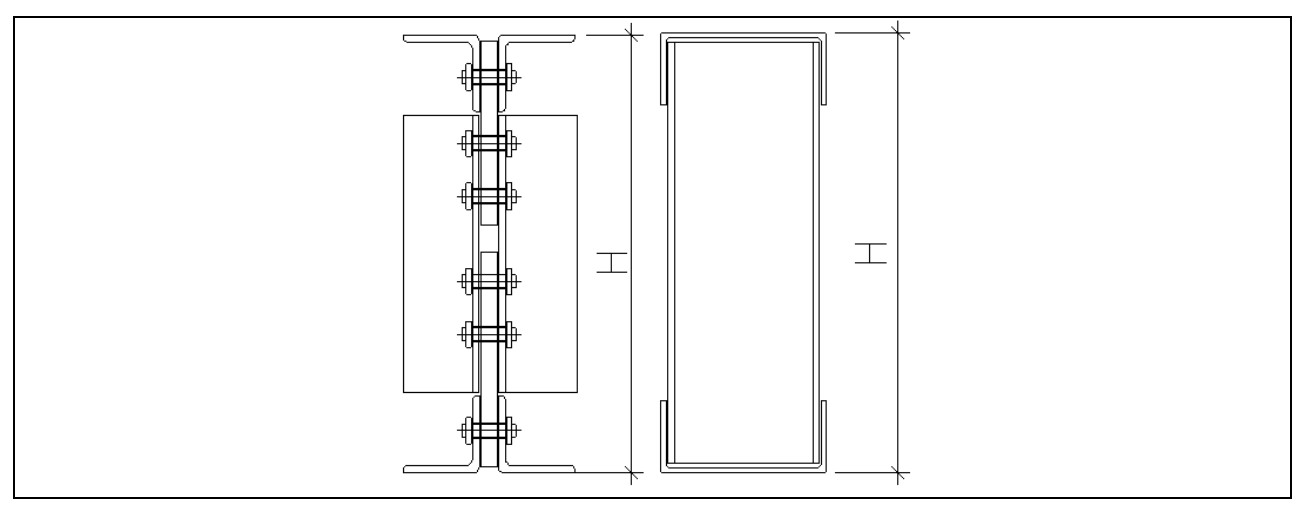

Figura 1 - Seção transversal dos perfis que compõem os modelos de treliças trapezoidais. Fonte: Adaptado de Rigo (2014). 


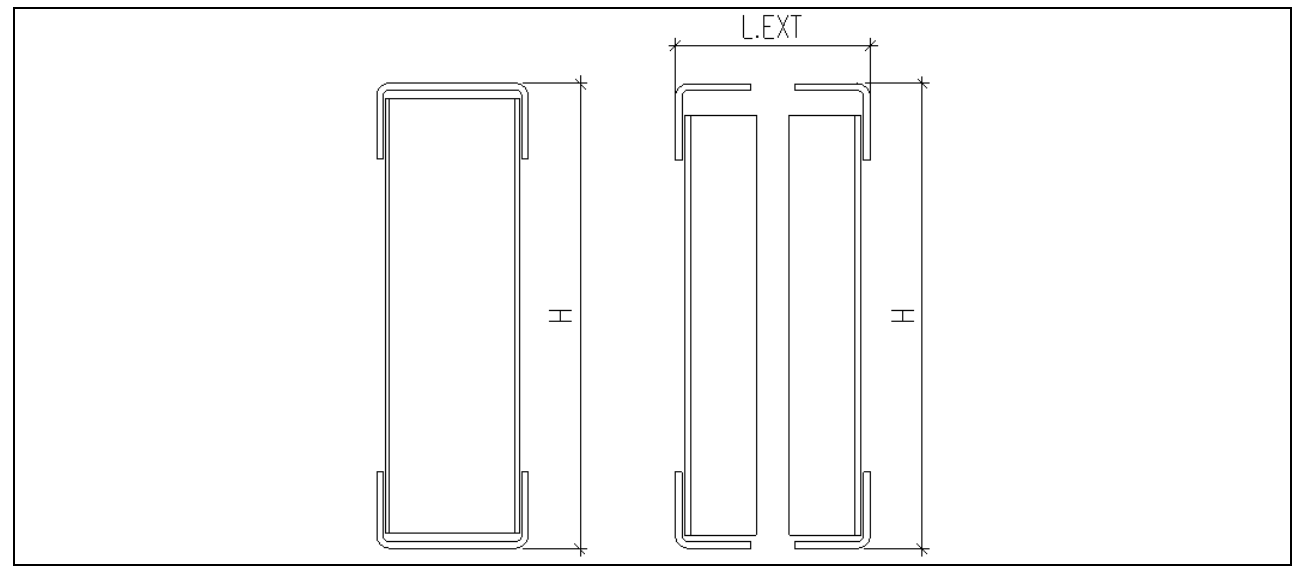

Figura 2 - Seção transversal dos perfis que compõem os modelos de treliças retangulares. Fonte: Adaptado de Rossoni (2015)

Nos modelos estruturais que foram analisados e dimensionados, foram definidos vários vãos dos pórticos, altura da coluna e distância entre pórticos conforme a configuração da figura 3 ( $a$ e b) e figura 4 e valores apresentados na Tabela 1 e 2:

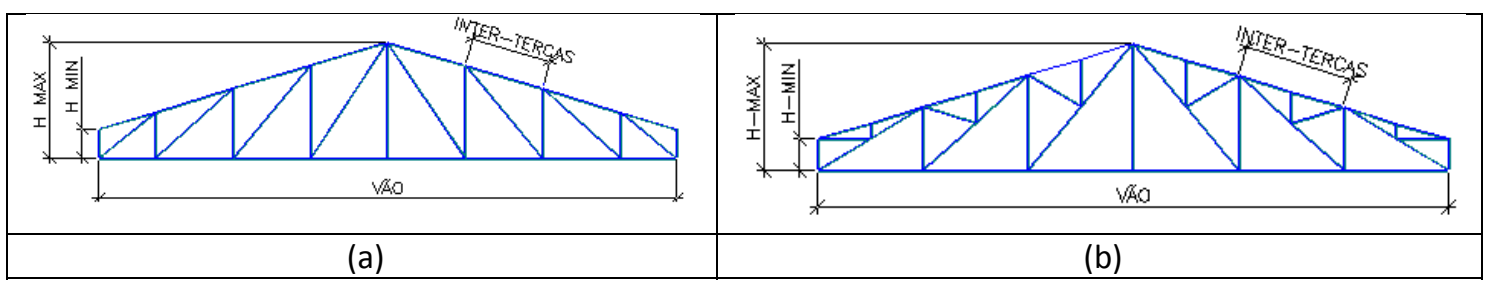

Figura 3 - Esquemas de composição das treliças plana trapezoidal de cobertura

Tabela 1 - Dimensões padrões para análise dos modelos de treliça plana trapezoidal

\begin{tabular}{|c|c|c|c|c|c|c|}
\hline $\begin{array}{c}\text { L-Vão } \\
\text { livre } \\
(\mathrm{m})\end{array}$ & $\begin{array}{c}\text { H - } \\
\text { Altura da } \\
\text { coluna } \\
(\mathrm{m})\end{array}$ & $\begin{array}{c}\text { B - Distância } \\
\text { entre } \\
\text { pórticos }(\mathrm{m})\end{array}$ & $\begin{array}{c}\text { Comprimento } \\
\text { da edificação } \\
(\mathrm{m})\end{array}$ & $\begin{array}{c}\text { H-MAX } \\
\text { Altura } \\
\text { máxima da } \\
\text { treliça }(\mathrm{m})\end{array}$ & $\begin{array}{c}\text { H-MIN } \\
\text { Altura mínima } \\
\text { da treliça }(\mathrm{m})\end{array}$ & $\begin{array}{c}\text { Inter-terças } \\
\text { Espaçamento } \\
\text { entre terças }(\mathrm{m})\end{array}$ \\
\hline 15 & 6 & 6 & 60 & 1,74 & 0,8 & 1,89 \\
\hline 25 & 9 & 9 & 63 & 2,36 & 0,8 & 1,80 \\
\hline 35 & 12 & 12 & 60 & 2,99 & 0,8 & 1,96 \\
\hline 45 & & & & 3,62 & 0,8 & 1,89 \\
\hline
\end{tabular}

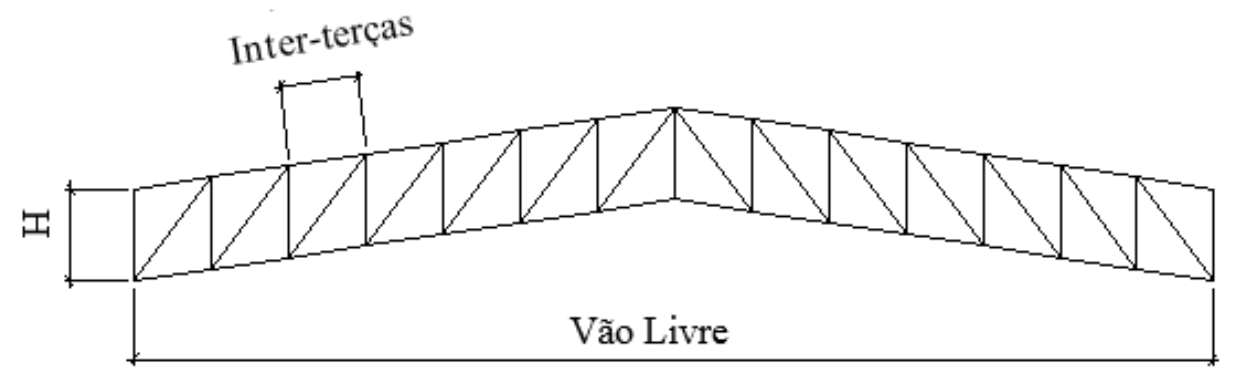

Figura 4 - Vista transversal da viga treliçada de cobertura retangular de banzos paralelos 
Tabela 2 - Dimensões padrões para análise dos modelos de treliça plana retangular de banzos paralelos. Fonte: Rossoni (2015)

\begin{tabular}{|c|c|c|c|c|c|}
\hline $\begin{array}{c}\text { L - Vão } \\
\text { livre }(\mathrm{m})\end{array}$ & $\begin{array}{c}\mathrm{H} \text { - Altura } \\
\text { da coluna } \\
(\mathrm{m})\end{array}$ & $\begin{array}{c}\text { B - Distância } \\
\text { entre pórticos } \\
(\mathrm{m})\end{array}$ & $\begin{array}{c}\text { Comprimento } \\
\text { da edificação } \\
(\mathrm{m})\end{array}$ & $\begin{array}{c}\text { H- Altura da } \\
\text { treliça }(\mathrm{m})\end{array}$ & $\begin{array}{c}\text { Inter-terças } \\
\text { Espaçamento entre terças } \\
(\mathrm{m})\end{array}$ \\
\hline 15 & 6 & 6 & 60 & 1,00 & 1,89 \\
\hline 25 & 9 & 9 & 63 & 1,75 & 1,80 \\
\hline 35 & 12 & 12 & 60 & 2,50 & 1,96 \\
\hline 45 & & & & 3,00 & 1,89 \\
\hline
\end{tabular}

Para análise estrutural dos pórticos e dimensionamento dos elementos de aço considerou-se as normas técnicas: ABNT NBR 6120:1980, ABNT NBR 6123:1986 ações devidas ao vento, ABNT NBR 8681:2003 segurança nas estruturas, ABNT NBR 8800:2008 projeto de estruturas com perfis laminados e soldados, ABNT NBR 14762:2010 projeto de estruturas com perfis formados a frio. Nessa análise para as ações e combinações foram consideradas as ações permanentes, incluído o peso próprio da estrutura, uma ação acidental mínima de $0,25 \mathrm{kN} / \mathrm{m}^{2}$, e o vento para velocidades básicas de $30,35,40$ e $45 \mathrm{~m} / \mathrm{s}$. As combinações utilizadas estão conforme aquelas previstas na ABNT NBR 8800:2008 e na ABNT 14762:2010 para estados-limites últimos e as frequentes para estados-limites de serviço. Além disso, foram verificadas as flechas dos elementos e das treliças.

Para exemplificar o uso dos ábacos propostos são apresentadas duas aplicações explicativas.

\section{RESULTADOS}

A partir da análise dos resultados obtidos para as seções da treliça trapezoidal, se observou que a maior diferença entre os perfis obtidos na verificação do dimensionamento é com relação ao vão maior $(45 \mathrm{~m})$ e em relação ao menor vão de $(15 \mathrm{~m})$, sendo que a variação de espessura foi pequena, pois aumentavam-se as dimensões da seção do perfil e reduzia-se a espessura.

Analisando as tabelas 3 e 4 dos perfis utilizados nas tesouras retangulares planas de banzos paralelos percebeu-se que as diferenças entre espessuras dos perfis para os galpões do menor vão para o maior, com as mesmas considerações de cálculo, não 
apresentam grande variação, mas foi o aumento nas dimensões do perfil o que repercutiu na não alteração da espessura.

As treliças retangulares planas de coberturas com banzos paralelos demonstraram ser muito funcionais, pois sua deformação ficou abaixo do limite de L/250, e também tendo espessura máxima de $7,94 \mathrm{~mm}$ em perfil $\mathrm{U}$ e $12,7 \mathrm{~mm}$ em cantoneira dupla, necessitando assim de equipamentos de menor capacidade para seu processo de produção.

A partir dos resultados do dimensionamento elaboraram-se ábacos para realizar o pré-dimensionamento da estrutura da cobertura em treliça plana trapezoidal e em treliça plana retangular. Esses ábacos foram elaborados considerando 4 (quatro) incógnitas, que são: a velocidade básica do vento, vão dos pórticos, espaçamento entre pórticos e pé-direito (altura da coluna).

Na montagem desses gráficos, primeiramente determina-se a união dos pontos de vão da treliça $(L)$ no eixo das abscissas eixo $(x)$ e o ponto da velocidade do vento $\left(V_{0}\right)$ no eixo das ordenadas $(\mathrm{y})$ conforme mostra a figura 5. Após termos o primeiro ponto que é a intersecção de $(L)$ com $\left(V_{0}\right)$ conforme figura 5 , gira-se o triângulo em $45^{\circ}$ no sentido horário e têm-se um segundo eixo de coordenadas (figura 6) e neste ábaco locase os pontos de espaçamento entre pórticos (B) no eixo das abscissas ( $\mathrm{x}$ ) e altura das colunas $(H)$ no eixo das ordenadas $(y)$. Com a união destes pontos determina-se o tipo de perfil a ser adotado para a treliça trapezoidal ou retangular. Através destes ábacos é possível também determinar a seção de pré-dimensionamento para o pilar para a condição de projeto desejada, mas essa consideração não será apresentada neste trabalho.

\subsection{Aplicação 1 - Treliça Plana trapezoidal}

Para dimensionar um pórtico com viga de cobertura treliçada trapezoidal com as seguintes características:

- vão do pórtico, largura: L=35,0m;

- velocidade do vento conforme Mapa de Isopletas: $V_{0}=45 \mathrm{~m} / \mathrm{s}$;

- espaçamento entre pórticos: $B=6,0 \mathrm{~m}$;

- altura da coluna: $\mathrm{H}=12,0 \mathrm{~m}$.

Pré-dimensionamento pelo Ábaco: 
- primeiramente encontra-se a largura de $\mathrm{L}=35 \mathrm{~m}$ no eixo horizontal $(\mathrm{x})$;

- no eixo vertical y, encontra-se a velocidade básica do vento $V_{0}=45 \mathrm{~m} / \mathrm{s}$, encontrando-se assim a primeira intersecção (figura 5);

- nesta interseção (figura 6) encontra-se um novo sistema de coordenadas com 9 opções, variando a altura da coluna $(H=6,9$ e $12 \mathrm{~m})$ e a distância entre pórticos ( $B=6,9$ e $12 \mathrm{~m})$, linhas estas que estão rotacionadas em $45^{\circ}$ a partir do eixo global horizontal da figura.

Rotacionando-se esta figura encontra-se os novos eixos globais a partir da origem da intersecção de $L$ e $V$, onde se encontra o ponto $H=12 m$ e $B=6 m$ (figura 7).

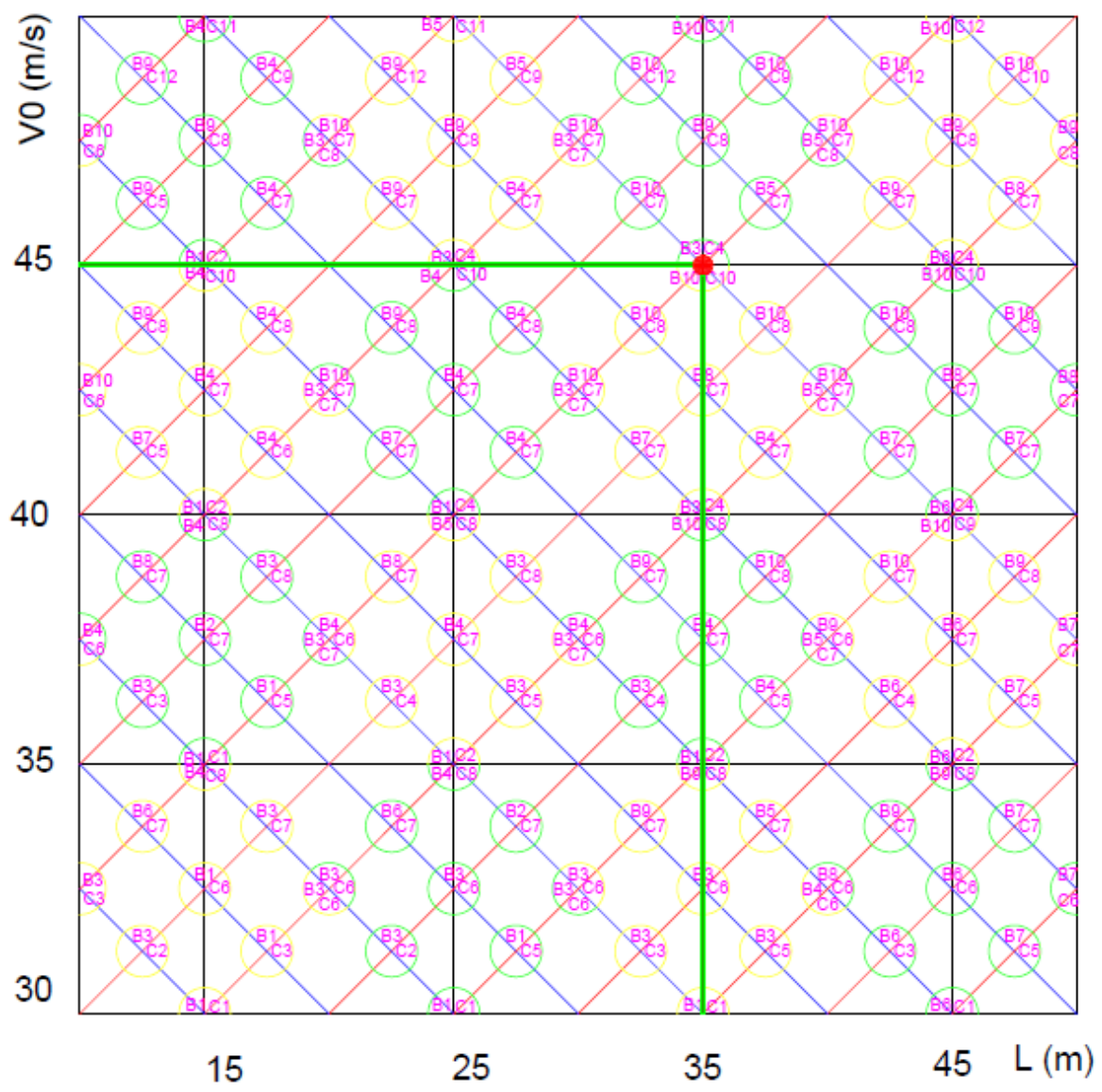

Figura 5 - Ábaco para definição de perfis de treliças planas trapezoidais com seção de perfis em cantoneira dupla. Fonte: Rigo (2014) 


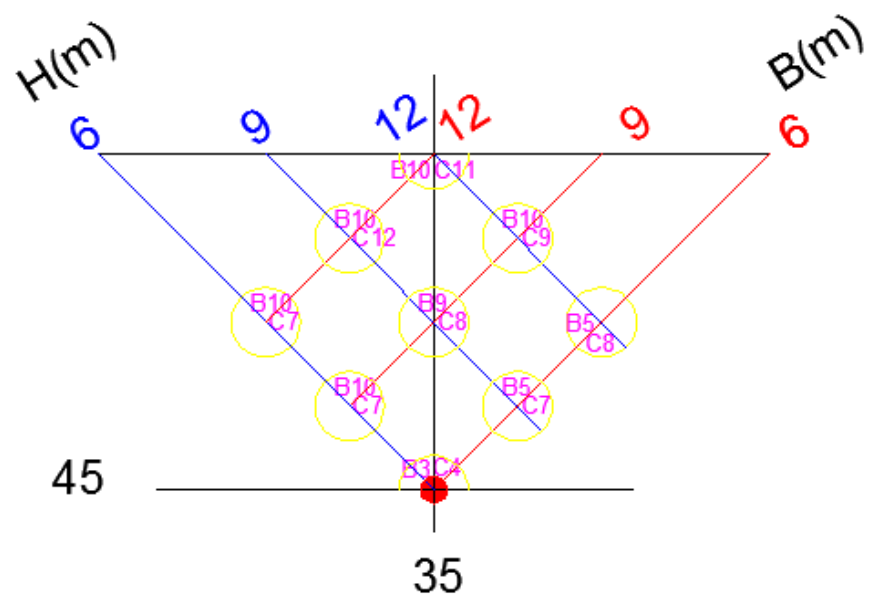

Figura 6 - Ponto de origem do novo sistema de coordenadas. Fonte: Rigo (2014)

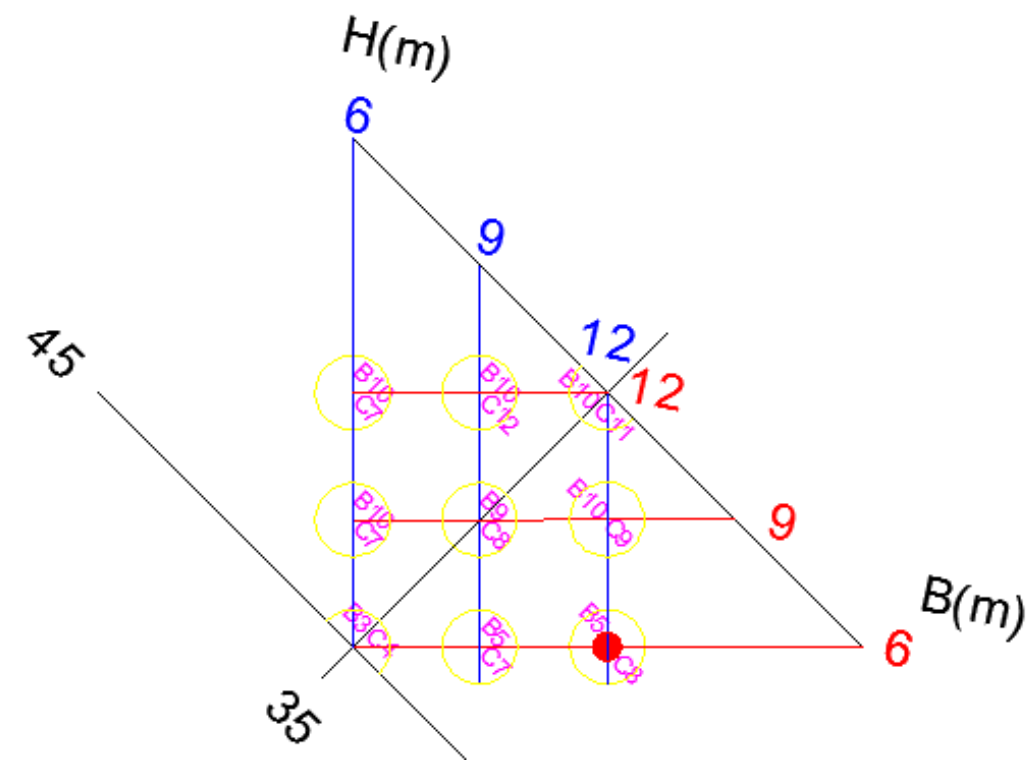

Figura 7 - Segundo eixo de coordenadas globais: Intersecção dos eixos $\mathrm{H}=12$ metros e $\mathrm{B}=6$ metros. Fonte: Rigo (2014)

Tabela 3 - Identificação do perfil por código do ábaco. Fonte: Rigo (2014)

\begin{tabular}{|c|c|c|}
\hline & Seção perfil dupla cantoneira (mm) & Seção perfil U (mm) \\
\hline B1 & $2 \mathrm{~L} 50 \times 2,25 \times 50$ & U $100 \times 50 \times 3$ \\
\hline B2 & $2 \mathrm{~L} 50 \times 2,65 \times 50$ & U $100 \times 75 \times 2,65$ \\
\hline B3 & $2 \mathrm{~L} 50 \times 3,75 \times 50$ & U $100 \times 75 \times 3$ \\
\hline B4 & $2 \mathrm{~L} 50 \times 4,75 \times 50$ & U $100 \times 75 \times 3,35$ \\
\hline B5 & $2 \mathrm{~L} 50 \times 6,35 \times 50$ & U $100 \times 75 \times 4,25$ \\
\hline B6 & $2 \mathrm{~L} 60 \times 2,65 \times 50$ & U $100 \times 75 \times 4,75$ \\
\hline B7 & $2 \mathrm{~L} 60 \times 3,35 \times 50$ & \\
\hline B8 & $2 \mathrm{~L} 60 \times 3,75 \times 50$ & \\
\hline B9 & $2 \mathrm{~L} 60 \times 4,75 \times 50$ & \\
\hline B10 & $2 \mathrm{~L} 60 \times 6,35 \times 50$ & \\
\hline
\end{tabular}


Portanto, conforme a figura 7 para a intersecção $H=12$ e B = 6, têm-se um código B5 que representa o perfil pré-dimensionado $2 \mathrm{~L} 50 \times 6,35 \times 50$ mostrado na tabela da tabela 3 para a treliça plana trapezoidal de cobertura com as características admitidas anteriormente, para $V_{0}=45 \mathrm{~m} / \mathrm{s}$ e $\mathrm{L}=35 \mathrm{~m}, \mathrm{~B}=6 \mathrm{~m}$ e H$=12 \mathrm{~m}$.

O mesmo procedimento de pré-dimensionamento deve ser realizado para treliças planas trapezoidais, porém compostas por perfis de seção transversal em $U$, utilizando o ábaco da figura 8 e seções disponíveis na tabela 3

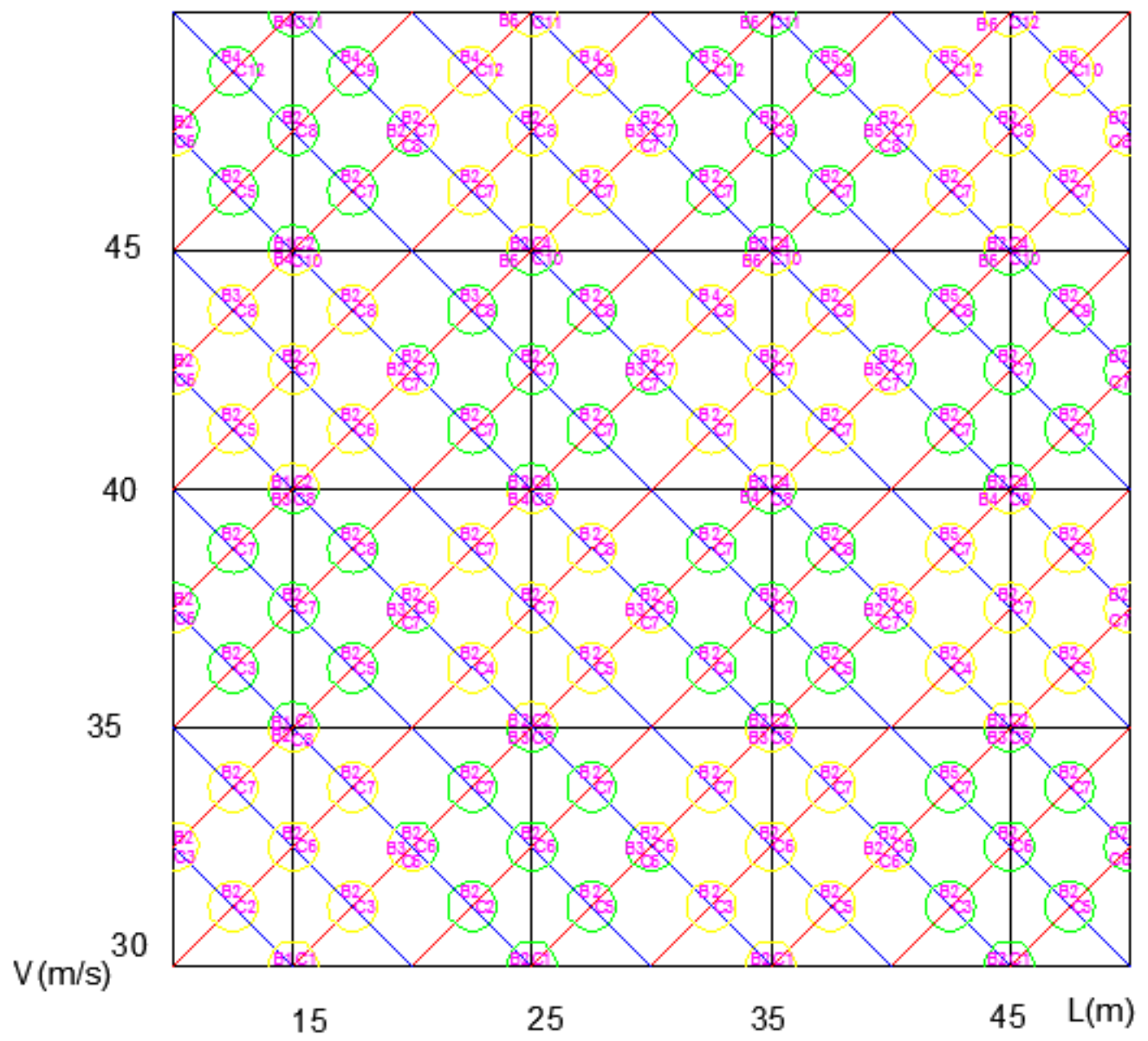

Figura 8 - Ábaco para definição de perfis de treliças planas trapezoidais com seção de perfis U. Fonte: Rigo (2014)

\subsection{Aplicação 2: Treliça Plana Retangular de Banzos Paralelos}

Para pré-dimensionar o perfil para viga de cobertura treliçada retangular de banzos paralelos, o processo é o mesmo do apresentado no exemplo 1, sendo:

- primeiramente encontrar o vão desejado no caso do exemplo de $\mathrm{L}=25 \mathrm{~m}$ no eixo de coordenadas cartesianas horizontal $(x), L(m)$; 
- após encontrar a velocidade básica do vento no caso desse exemplo $\mathrm{V}=40 \mathrm{~m} / \mathrm{s}$ no eixo de coordenadas cartesianas vertical " $\mathrm{y}$ ", em $\mathrm{m} / \mathrm{s}$, encontra-se a primeira intersecção no eixo das coordenadas cartesianas representada na figura 9;

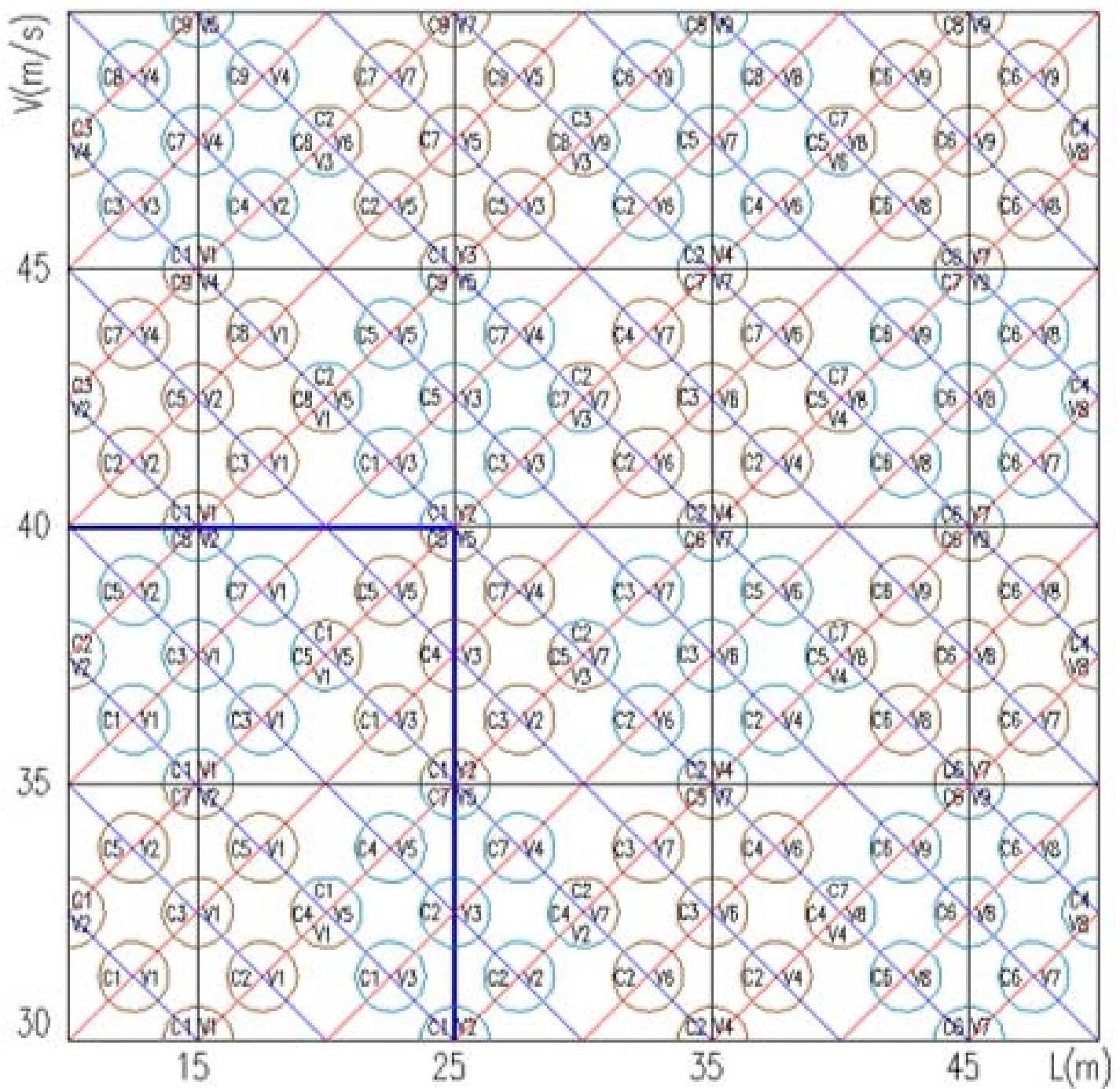

Figura 9 - Ábaco para definição de perfis de treliças planas retangulares com seções de perfis U. Fonte: Rossoni (2015)

- esta intersecção encontra-se o ponto de origem do novo sistema de coordenadas globais com 9 opções, variando o pé-direito ( $H=6,9$ e 12m) e a distância entre pórticos $(B=6,9$ e $12 \mathrm{~m})$, linhas estas que estão rotacionadas em $45^{\circ}$ e $135^{\circ}$ a partir do eixo global (figura 10); 


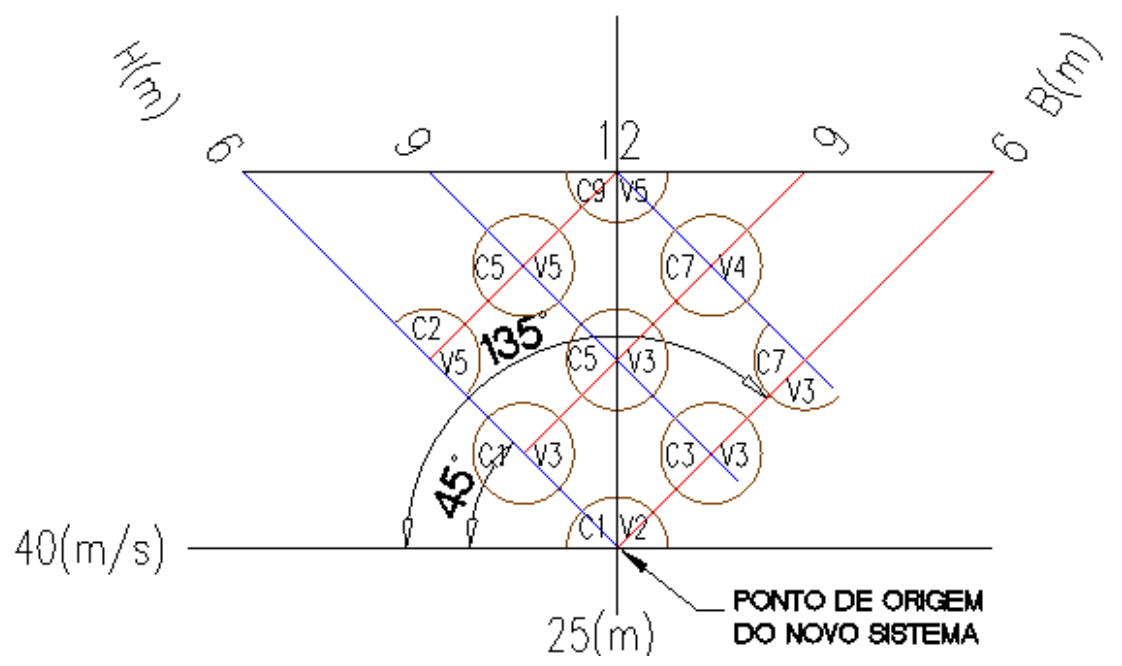

Figura 10 - Ponto de origem do novo sistema de coordenadas. Fonte: Rossoni (2015)

- encontra-se então a intersecção entre o eixo que representa o pé-direito, $\mathrm{H}=9 \mathrm{~m}$ e o eixo que representa o espaçamento entre pórticos, $\mathrm{B}=12 \mathrm{~m}$ (figura 11).

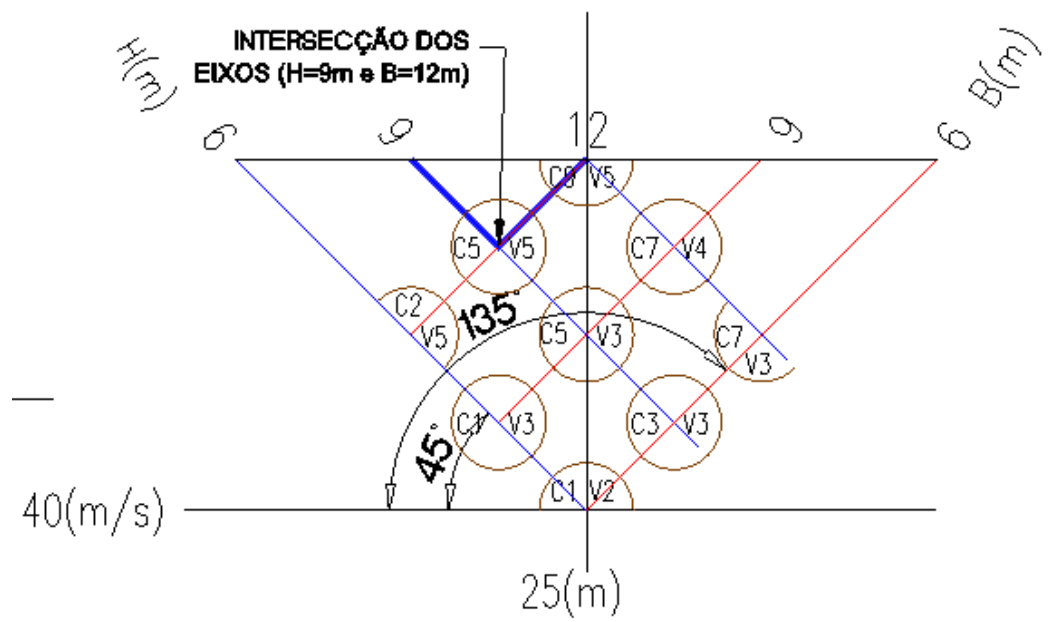

Figura 11 - Segundo eixo de coordenadas globais: Intersecção dos eixos $\mathrm{H}=9$ metros e $\mathrm{B}=12$ metros. Fonte: Rossoni (2015)

Esta intersecção (figura 11) define o ponto no qual se encontra o perfil prédimensionado para as treliças de cobertura planas retangulares para as considerações anteriormente previstas, para $L=25 \mathrm{~m}, \mathrm{~V}_{0}=40 \mathrm{~m} / \mathrm{s}, \mathrm{H}=9 \mathrm{~m}$ e $\mathrm{B}=12 \mathrm{~m}$. Com isso o perfil requerido para essa combinação do exemplo 2 corresponde ao código V5 que representa o perfil pré-dimensionado (U 200x80x6,35) da tabela 4.

O mesmo processo de pré-dimensionamento deve ser realizado para treliças planas retangulares, porém compostas por perfis de seção transversal em dupla 
cantoneira, utilizando o ábaco da figura 12 e seções com seu respectivo código do ábaco, disponíveis na tabela 4.

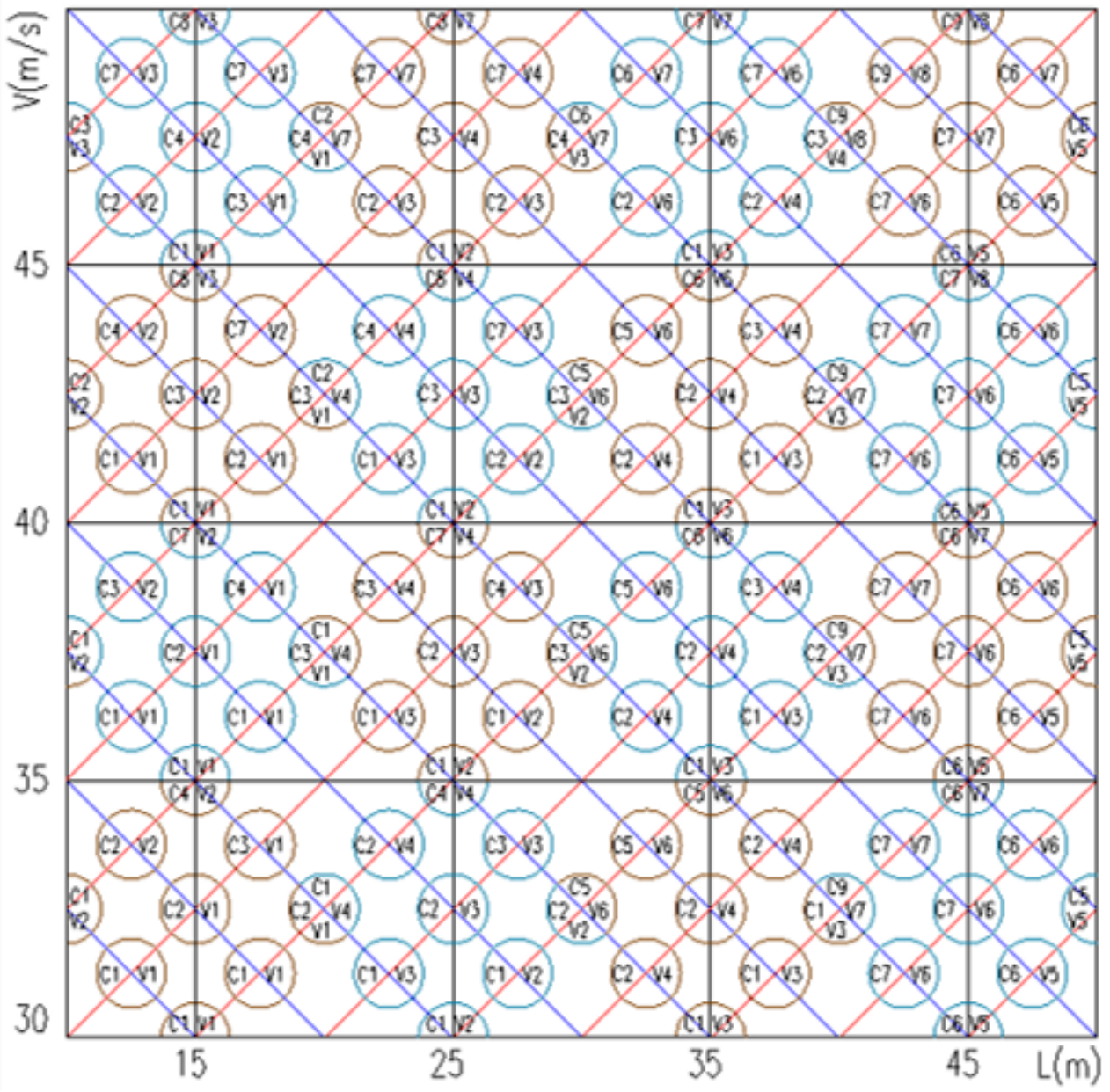

Figura 12 - Ábaco para definição de perfis de treliças planas retangulares com seções de dupla cantoneira. Fonte: Rossoni (2015)

Tabela 4 - Identificação do perfil por código do ábaco. Fonte: Rossoni (2015)

\begin{tabular}{|c|c|c|c|}
\hline & Treliça - Perfil U & \multicolumn{2}{|c|}{ Treliça - Perfil Dupla cantoneira } \\
\hline & Seção perfil (mm) & Seção perfil (mm) & Lado externo (mm) \\
\hline V1 & U $150 \times 70 \times 3,75$ & $2 \mathrm{~L} 75 \times 3,75 \times 75$ & 200 \\
\hline V2 & U 150x70x4,75 & $2 \mathrm{~L} 75 \times 4,75 \times 75$ & 200 \\
\hline V3 & U $200 \times 80 \times 4,75$ & $2 \mathrm{~L} 75 \times 6,35 \times 75$ & 200 \\
\hline V4 & U 200x100x4,75 & $2 \mathrm{~L} 75 \times 9,52 \times 75$ & 200 \\
\hline V5 & U $200 \times 80 \times 6,35$ & $2 \mathrm{~L} 100 \times 6,35 \times 100$ & 250 \\
\hline V6 & U $200 \times 100 \times 6,35$ & $2 \mathrm{~L} 100 \times 7,94 \times 100$ & 250 \\
\hline V7 & U $250 \times 150 \times 6,35$ & $2 \mathrm{~L} 100 \times 9,52 \times 100$ & 250 \\
\hline V8 & U $300 \times 150 \times 6,35$ & $2 \mathrm{~L} 125 \times 12,7 \times 125$ & 300 \\
\hline
\end{tabular}




\section{CONCLUSÕES}

As diversas soluções estruturais analisadas neste trabalho tiveram por objetivo apresentar sugestões de pré-dimensionamento de seções para diversos vãos e duas configurações de treliças. As prescrições das normas ABNT NBR 8800:2008 e ABNT NBR 14762:2010 foram seguidas e foram apresentados os ábacos para serem usados com dois exemplos de uso.

O uso destes ábacos facilitará a estudantes em cursos de graduação e os profissionais iniciantes a realização de projetos de coberturas de aço.

\section{AGRADECIMENTOS}

À Stabile Engenharia pela licença do programa MCalc 3D concedida para a realização da pesquisa.

\section{REFERÊNCIAS}

ASSOCIAÇÃO BRASILEIRA DE NORMAS TÉCNICAS (ABNT). NBR 6120:1980 Cargas para o cálculo de estruturas para edificações. Rio de Janeiro: 1980.

NBR 14672:2010 Dimensionamento de estruturas de aço constituídas por perfis formados a frio. Rio de Janeiro: 2004.

. NBR 6123:1988. Forças devidas ao vento em edificações. Rio de Janeiro: 1988.

NBR 6355:2012. Perfis Estruturados de aço formados a frio: padronização. Rio de Janeiro: 1988.

NBR 8681:2003 Ações e segurança nas estruturas - procedimento. Rio de Janeiro: 2003

NBR 8800:2008. Projetos de Estruturas de Aço e de Estruturas Mistas de Aço e Concreto de Edifícios. Rio de Janeiro: 2008.

BIANCHI, Pollyana; NOVELLO, Marinês Silvani; PRAVIA, Zacarias Chamberlain; Um ábaco para pré-dimensionamento de seções de coberturas em arco treliçadas de perfis formados a frio. Associação Brasileira da Construção Metálica -ABCEM. São Paulo, Edição 119, p. 422 a 45, dez. 2015. 
BIANCHI, Pollyanna Fernandes. Pré-dimensionamento de coberturas sustentáveis em arco treliçadas compostas por perfis de aço conformados a frio. Universidade de Passo Fundo, Passo Fundo, 2014.

CARVALHO, Paulo Roberto M. de.; GRIGOLETTI, Gladimir.; DALTROZO BARBOSA, Giovana. Curso Básico de perfis de aço formados a frio. 3ạ edição. Porto Alegre [s.n.], 2014. 370 p.

CHAMBERLAIN PRAVIA, Zacarias. M., Drehmer, G. A., Galpões para usos gerais. 4ạ ed. Instituto Aço Brasil. Rio de Janeiro: IAB/CBCA, 2010. 74p.

D’ÁLAMBERT, Flávio Correa. Galpão em pórticos com perfis estruturais laminados. Instituto Brasileiro de Siderurgia / Centro Brasileiro da Construção em aço. Rio de Janeiro, 5a ed. 2014. 68p.

REBELLO, Yopanan Conrado Pereira. Bases para projeto estrutural na arquitetura. 5a ed. São Paulo: Zigurate, 2007. 286 p.

RIGO, Judiclar. Pré-dimensionamento de coberturas sustentáveis treliçadas em perfis de aço dobrados a frio. Universidade de Passo Fundo, Passo Fundo, 2014.

ROSSONI, Cristiano. Pré-dimensionamento de coberturas sustentáveis em arcos e treliças planas em perfis dobrados a frio e perfis tubulares. Universidade de Passo Fundo, Passo Fundo, 2015.

STABILE ENGENHARIA LTDA. Manual Mcalc3D. 3a versão. 